\title{
VIENT DE PARAîTRE
}

STRAUSS, LEO. Qu'est-ce que la philosophic politique? Traduit de l'anglais par Olivicr Sedcyn. Paris: Presses Universitaires de France, 1992.

"Le titre de ce recucil vient de celui de la premicre étude publiée dans cet ouvrage, qui lui donne ainsi son sens immédiat. Mais il faut souligner immédiatement la singularité du regard que Leo Strauss porte sur notre tradition de philosophie politique. Il ne s'agit pas de philosophie politique au sens d'une branche "locale" de la philosophie; d'une certaine manière, la philosophie politique est "philosophie premiere" et dans cette mesure la philosophie est éminemment problématique, elle engage toute la philosophie, c'est-à-dire tous les problemes fondamentaux de la vie humaine. Les éludes réunies dans ce volume sont pour les unes générales. Strauss traite d'abord de la définition de la philosophie politique et de son histoire en soulignant la nécessité de se pencher sérieusement sur les penseurs de la philosophie politique classique; puis il étudie quelques moments privilégiés de l'histoire de la philosophie politique (Fârâbi, Maimonide, Hobbes, Locke); il traite ensuite de l'art d'écrire oublie et d'un homme remarquable (Kurt Riezler), qui fut à la fois un connaisseur profond de la réalité politique internationale et un philosophe contemporain, en des pages surprenantes de liberté et de profondeur. Car la philosophie politique ici développée est fondamentalement soucicuse de ce qu'il y a de plus élevé et de plus profondément humain dans l'homme."

Olivier Sedeyn

CLARK, S. H. Paul Ricoeur. London and New York. Routledge, 1990.

"No contemporary thinker has participated more fully in the intellectual debates of the post-war period than Paul Ricoeur. Ricoeur's writings 
range from an initial concern with existentialism through structuralism, psychoanalysis and hermeneutics, to his now classic studics of metaphor and narrative. Stephen Clark's introduction to Ricoeur's work is the first to survey the entire range of his writings and to situate them within the context of contemporary post-structuralism. It includes an extended discussion of Ricoeur's most recent project, Time and Narrative.

Stephen Clark is British Academy Research Fellow at Queen Mary and Westfield College, University of London."

ROZENBERG, JACQUES J. Bio-cognition de l'individualite: Philosophèmes de la vie et du concept. Paris: Presses Universitaires de France, 1992.

"Cet ouvrage thématise la notion d'individualité à travers les rapports, à la fois agonistes et antagonistes, que l'histoire de la philosophie a entretenues avec celle des sciences de la vie. Il vise d'abord à ressaisir l'idée de vie d'un point de vue logique et philosophique, en mettant au jour l'enracinement biologique de toute détermination conceptuelle. Il confronte ensuite un certain nombre de philosophèmes à la recherche scientifique effective. Profondément unitaire, cette double demarche propose une analyse systématique du concept d'indiviiduation et des apories dont il a fait l'objet d'Aristote a Hegel. Se trouvent ainsi dégagées les caractéristiques opératoires et cognitives de l'individualité vivante, permettant d'une part d'éclairer de façon originale certains présupposés de la philosophie occidentale, et d'autre part de préfigurer une nouvelle approche pragmatique. Celle-ci paraît susceptible de fonder un continuum psychologique, capable d'intégrer les réflexions métapsychologique et psychopathologique." 